\title{
Design and Performance Analysis of Multi-scale NOMA for Future Communication-Positioning Integration System
}

\author{
Lu Yin, Member, IEEE, Jiameng Cao, Qiang Ni, Senior Member, IEEE, Yuzheng Ma, Song Li, Member, IEEE
}

\begin{abstract}
This paper presents a feasibility study of a novel multiple access technique called Multi-Scale NonOrthogonal Multiple Access (MS-NOMA) for the next generation communication-positioning integration system. Different from the traditional positioning signals which are mostly Time Division Multiple Access with communication signals and are broadcast to all users, MS-NOMA supports continuous positioning waveform and flexible configurations for different positioning users to obtain higher ranging accuracy, lower positioning latency, less resource consumption and better signal coverage. Our major contributions are: Firstly, we present the MS-NOMA waveform and evaluate its performances by theoretical and simulation analyses. The results show it is feasible to use the MS-NOMA waveform to achieve high positioning accuracy and low Bit Error Rate with little resource consumption simultaneously. Secondly, to achieve optimal positioning accuracy and signal coverage, we model the power allocation problem for MS-NOMA as a convex optimization problem satisfying the Quality of Services requirement and other constraints. Then, we propose a novel Communication and Positioning Performances constrained Positioning Power Allocation (CP4A) algorithm which allocates the power of all P-Users iteratively. The theoretical and numerical results show our proposed MS-NOMA waveform with CP4A algorithm has great improvements of ranging/positioning accuracy than traditional Positioning Reference Signal in cellular network.
\end{abstract}

Index Terms-MS-NOMA, communication-positioning integration, positioning, power allocation.

\section{INTRODUCTION}

Nowadays, the Location Based Services (LBS) are growing rapidly and attracting much attention with the proliferation of mobile devices [1]. Besides the well known Global Navigation Satellite Systems (GNSS) which can only be used in open areas as their signals are easily blocked or interfered by buildings [2], communication-based positioning methods $(5 \mathrm{G}$, Bluetooth, Wi-Fi, etc.) have been greatly developed in recent years as the ready-made communication networks have natural

This research was supported in part by the National Natural Science Foundation of China under Grant 61801041 and in part by the Beijing Municipal Natural Science Foundation under Grant L191003. (Corresponding author: Lu Yin.)

$\mathrm{Lu}$ Yin, Jiameng Cao, and Yuzheng Ma are with the School of Electronic Engineering, Beijing University of Posts and Telecommunications, Beijing, 100876, China, e-mail: inlu_mail@bupt.edu.cn, caojiameng@bupt.edu.cn, mayuzhengjoy@gmail.com.

Qiang Ni is with the School of Computing and Communications, Lancaster University, InfoLab21, LA1 4WA, U.K. e-mail: q.ni@lancaster.ac.uk.

Song $\mathrm{Li}$ is with the School of Information and Control Engineering, China University of Mining and Technology, Xuzhou, China. e-mail: lisong@cumt.edu.cn. advantages of wide coverage, easy deployment and low-cost [3], [4], [5].

There are mainly two categories of communication-based positioning: 1) Based on the existing communication components, such as positioning by fingerprint matching or Sounding Reference Signal (SRS) [4], [6]; 2) Based on the redesigned new waveform, such as positioning by Positioning Reference Signal (PRS) in cellular network or Constant Tone Extensions (CTE) in Bluetooth Low Energy 5.1 (BLE 5.1) [7], [8]. The positioning performance of the $1^{\text {st }}$ category is always unsatisfactory as the communication system is not designed for positioning purpose specifically. Better performance is usually obtained by the $2^{\text {nd }}$ category because of the exclusive positioning waveform.

In the upcoming B5G (beyond-5G) and 6G eras, emerging industries will demand higher positioning requirements. For example, higher positioning accuracy, measuring frequency and reliability should be satisfied in the scenarios like automatic driving and industrial equipment/robotic control [9], [10]. However, most of the traditional methods are just combinations of communication and positioning components by Time Division Multiple Access (TDMA) and often neglect their interference. For example, PRS will occupy a great number of resource blocks to obtain better positioning performance. While communication performance will degrade significantly without these resource blocks. Even so, PRS can not meet the accuracy and latency requirements simultaneously [11]. It is because the discontinuous PRS is hardly tracked which leads to low ranging accuracy, and there is a long interval between two PRS frames which leads to low measuring frequency [12]. On the other hand, to obtain higher measuring accuracy, positioning components should be transmitted continuously from different base stations (BS). Then, severe near-far effect between these positioning components make the signals from far BSs more difficult to be accessed [11], [13]. Consequently, poor geometric distribution of BSs is achieved which further worsens the positioning accuracy and reliability [14].

To this end, a novel communication-positioning integration waveform has to be designed which must satisfy the following requirements:

1) The interference between the positioning and communication components must be weak enough. i.e. The positioning component must consume as few communication resources as possible to ensure the Quality of Services $(\mathrm{QoS})$ requirements of communication. Meanwhile, the 
positioning performance should be good enough to satisfy the future requirements under massive communication resources.

2) The positioning components should be continuous. i.e. The receiver can track the signal for better measurement accuracy and higher measuring frequency.

3) In the down-link use case, the positioning components for different users must be configurable to reduce the near-far effect. i.e. Different users should obtain different signal strengths or gains from the same BS.

To satisfy the above requirements, we propose a novel multiple access technique for the next generation communicationpositioning integration system, called Multi-Scale NonOrthogonal Multiple Access (MS-NOMA), which superposes a significant low-power positioning component to the communication one without much interference based on the NOMA principle [15], [16]. In time domain, the MS-NOMA waveform is modulated by the pseudorandom noise sequence to obtain spreading gains and ensure a continuous transmission. In frequency domain, Orthogonal Frequency Division Multiplexing (OFDM) is employed for different positioning users as Fig. 1 shows.

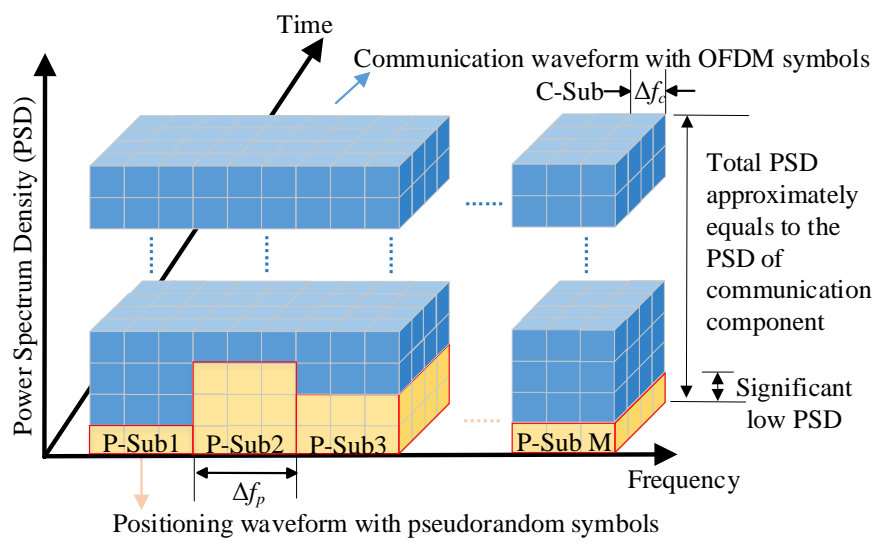

Fig. 1. The MS-NOMA architecture

In the proposed MS-NOMA waveform, $\Delta f_{c}$ and $\Delta f_{p}$ represent the sub-carrier spacing of communication and positioning waveform, respectively. For the maximum spectrum effectiveness, they are designed as $\Delta f_{p}=G \Delta f_{c}, G \in \mathbb{N}_{+}$. Define $B$ as the total bandwidth, then there are maximum $N=B / \Delta f_{c}-1$ and $M=B / \Delta f_{p}-1$ sub-carriers for communication and positioning purpose, respectively. For clear representation, define C-Sub/P-Sub and C-User/P-User as the abbreviations of communication/positioning sub-carrier and communication/positioning user, respectively. Notice each P-Sub may transmit to a single P-User or many P-Users who have similar channel states and positions. To simplify the problem and without any loss of generality, we assume each $\mathrm{C}-\mathrm{Sub} / \mathrm{P}-\mathrm{Sub}$ is occupied by a C-User/P-User. And all C-Subs have the same power. Then, to acquire the highest positioning accuracy over the whole network under the hearability and QoS requirements, the parameters of different P-Subs should be allocated carefully. In this paper, we focus on the power allocation of the P-Subs and assume other parameters are constant. It is worth noting that MS-NOMA waveform has the potential to allocate all kinds of resources for better performances of both positioning and communication, such as the power, the bandwidth, the spreading code, etc.

In a conventional OFDM system, it is proved that waterfilling over the sub-carriers is the optimal power allocation strategy [17]. However, it does not consider the interference between different types of users. In [18], where the second user transmits over spectrum holes left in the primary system, an optimal power allocation strategy is proposed. They maximize the down-link capacity of the second user by remaining the interference introduced to the primary user within a tolerable range. In the NOMA system, the power allocation is mostly investigated for signal demodulation and relay transmission [19], [20]. But these algorithms can not be used in our problem that has different models which is more complicated.

To the best of our knowledge, it is the first time that proposes a methodology which employs configurable waveform to different P-Users by allocating their power in order to improve the coverage and accuracy performances which is an essential difference with the traditional positioning methods. Because there are few studies about the waveform design and power allocation algorithm of communication-positioning integration system, it makes our study very challenging. A preliminary part of this study was presented in a letter paper [21] which focuses on the basic characteristics of MS-NOMA based on single-cell scenario. In this journal version, as compared to [21], we study realistic positioning problem based on multicell scenario and carry out detailed designs by conducting more in-depth mathematical analyses. The main contributions of this paper are:

1) We present the positioning model by the proposed MSNOMA waveform and analyze the limitation of positioning by other existing signals.

2) We analyze the interference between the communication and positioning components of the MS-NOMA waveform in the multi-cell scenario. Bit Error Rate (BER) for communication and the ranging accuracy for positioning are derived.

3) We model the power allocation problem for the MSNOMA waveform as a convex optimization problem. It minimizes the average positioning error variance of all P-Users in the network by considering the QoS, the power budget and the hearability requirements. To solve this problem, we propose a novel Communication and Positioning Performances constrained Positioning Power Allocation (CP4A) algorithm which allocates the power of all P-Subs iteratively and derive its closed solution.

4) A series of theoretical and numerical analyses are done to evaluate the performances of positioning by MS-NOMA waveform. The results show our proposed MS-NOMA waveform has a great improvement of ranging/positioning accuracy than traditional PRS, and reduces the resource consumption dramatically which means both positioning and communication requirements could be satisfied simultaneously.

Notations: Subscript $p$ and $c$ represent the positioning and 
communication, respectively. $\|\cdot\|$ represents the Euclidean distance. The operator $\operatorname{cov}(\cdot)$ represents the covariance. $\mathcal{M}, \mathcal{N}$, $\mathcal{K}$ and $\mathcal{K}^{k}$ represent the set $\{1, \ldots, M\},\{1, \ldots, N\},\{1, \ldots, K\}$ and $\{1, \ldots, k-1, k+1, \ldots, K\}$,respectively.

\section{SySTEM MODEL}

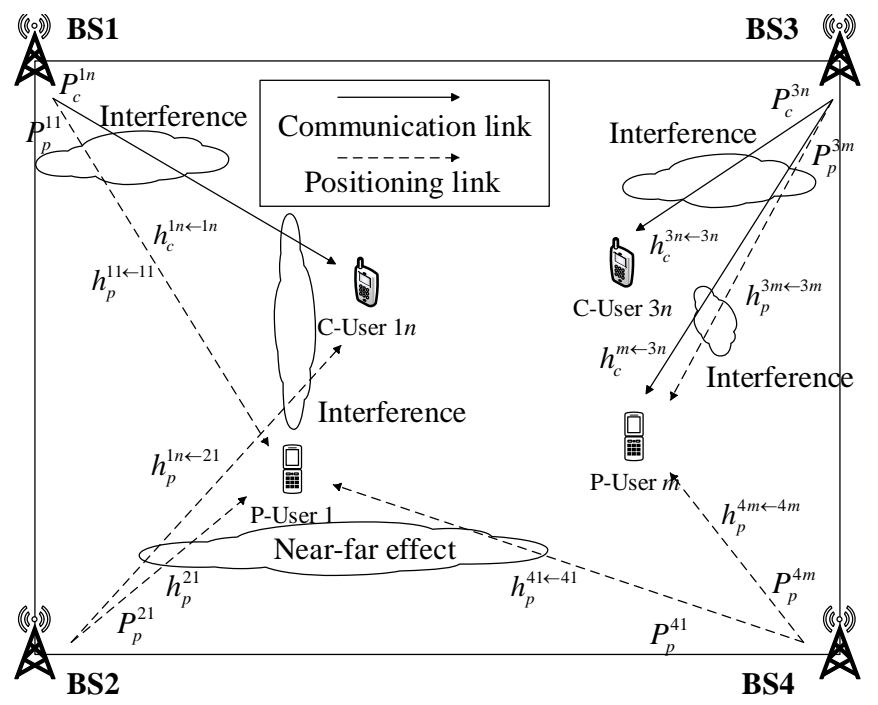

Fig. 2. System model of positioning by the MS-NOMA waveform

Consider a typical positioning scenario in spatial domain with $K$ BSs as Fig. 2 shows. C-Users and P-Users may locate at different positions. Let us define $h^{k y \leftarrow k x}$ as the instantaneous channel gain of sub-carrier $k x$ received by the user $k y$ which is known by the BSs through a delay- and error-free feedback channel. Then, the instantaneous channel gains of MS-NOMA waveform in typical positioning scenario are listed in Table I. Please notice each C-User connects only one BS and P-User connects multiple BSs, so the indexes of the two kinds of users are $k n$ and $m$, respectively.

TABLE I

INSTANTANEOUS CHANNEL GAINS OF MS-NOMA WAVEFORM IN TYPICAL POSITIONING SCENARIO

\begin{tabular}{|c|c|}
\hline $\begin{array}{l}\text { Instantaneous } \\
\text { channel gain }\end{array}$ & Definition \\
\hline$h_{c}, h_{p}$ & $\begin{array}{l}\text { Instantaneous channel gains of communication and } \\
\text { positioning waveform, respectively. }\end{array}$ \\
\hline$h_{c}^{k n \leftarrow k n}$ & $h_{c}$ of sub-carrier $k n$ received by the C-User $k n$ \\
\hline$h_{p}^{m \leftarrow k m}$ & $h_{p}$ of sub-carrier $k m$ received by the P-User $m$. \\
\hline$h_{c}^{m \leftarrow k n}$ & $h_{c}$ of sub-carrier $k n$ received by the P-User $m$. \\
\hline$h_{p}^{k n \leftarrow k^{\prime} m}$ & $h_{p}$ of sub-carrier $k^{\prime} m$ received by the C-User $k n$. \\
\hline$h_{p}^{m \leftarrow k_{k}^{\prime} m}$ & $\begin{array}{c}h_{p} \text { of the strongest sub-carrier except the P-Sub } \\
k m \text { received by the P-User } m .\end{array}$ \\
\hline$h_{c}^{m \leftarrow k^{\prime}}$ & See (62). \\
\hline
\end{tabular}

If the BSs are perfectly synchronized, the P-Users will use time-based algorithm to estimate their locations. Then, the horizontal positioning accuracy of P-User $m$ can be expressed as

$$
\Psi^{m}=\sqrt{\sum_{k \in \mathcal{K}}\left(\lambda^{k m} \sigma_{\rho}^{k m}\right)^{2}}
$$

where $\lambda^{k m}$ represents the geometric-dilution and $\sigma_{\rho}^{k m}$ represents the ranging error variance. Detailed derivation of (1) can be found in Appendix A. Please notice that $\Psi^{m}$ represents the lower bound of positioning error as well.

\section{FEATURES OF MS-NOMA WAVEFORM}

\section{A. Interference of Positioning to Communication}

In order to evaluate the interference of positioning to communication more accurately, we assume that the inter-cell interference between the communication components can be ideally eliminated [22]. Then, the BER of C-Sub $k n$ is

$$
B E R^{k n}=\Gamma \operatorname{erfc}\left(\frac{\gamma\left|h_{c}^{k n \leftarrow k n}\right|^{2} P_{c} T_{c}}{I^{k n}+2 N_{0}}\right)
$$

where $\Gamma$ and $\gamma$ are determined by the modulation and coding schemes [23]. $P_{c}$ is the power of communication component. $T_{c}$ is the period of communication symbol. $N_{0}$ is the singlesided Power Spectral Density (PSD) of the environment noise. $I^{k n}$ represents the interference of the positioning components to the C-User $k n$. Notice that the power of the positioning components from different BSs may be similar by power allocation, the interference caused by the positioning components from the neighbor BSs can not be ignored. Then, we have

$$
I^{k n}=\sum_{k^{\prime} \in \mathcal{K}} \sum_{m \in \mathcal{M}} \bar{P}_{p}^{k n \leftarrow k^{\prime} m}
$$

where $\bar{P}_{p}^{k n \leftarrow k^{\prime} m}$ is the power of the P-Sub $k^{\prime} m$ received by C-User $k n$ which satisfies

$$
\begin{aligned}
\bar{P}_{p}^{k n \leftarrow k^{\prime} m} & =\left|h_{p}^{k n \leftarrow k^{\prime} m}\right|^{2} P_{p}^{k^{\prime} m} G_{p}^{m}\left(n \Delta f_{c}\right) \\
& =\left|h_{p}^{k n \leftarrow k^{\prime} m}\right|^{2} P_{p}^{k^{\prime} m} T_{p} \operatorname{sinc}^{2}\left(m-\frac{n}{G}\right)
\end{aligned}
$$

where $P_{p}^{k^{\prime} m}$ is the power of P-Sub $k^{\prime} m . G_{p}^{m}(f)$ is the normalized PSD of P-Sub $m$ which satisfies

$$
G_{p}^{m}(f)=T_{p} \operatorname{sinc}^{2}\left[\left(f-m \Delta f_{p}\right) T_{p}\right]
$$

where $T_{p}$ is the period of the positioning symbol.

\section{B. Ranging Accuracy of MS-NOMA waveform}

The receiver could use a Delay Locked Loop (DLL) to track the positioning component. Taking the coherent earlylate discriminator for example [24], the tracking/ranging accuracy of the P-Sub $\mathrm{km}$ can be written as (6) shows. Where $a=B_{L}\left(1-0.5 B_{L} T_{c o h}\right)$ is determined by the loop noise bandwidth $B_{L}$ and the coherent integration time $T_{c o h} . B_{0}$ is the central frequency of MS-NOMA waveform. $B_{f e}$ is the double-sided front-end bandwidth. $D$ is the early-late spacing of DLL. $G_{s}^{m}(f)$ is the PSD of the communication components received by $\mathrm{P}$-User $m$ which satisfies

$$
G_{s}^{m}(f)=\sum_{k^{\prime} \in \mathcal{K}} \sum_{n \in \mathcal{N}}\left|h_{c}^{m \leftarrow k^{\prime} n}\right|^{2} P_{c} G_{c}^{n}(f)
$$




$$
\left(\sigma_{\rho}^{k m}\right)^{2}=\frac{a \int_{B_{0}-B_{f e} / 2}^{B_{0}+B_{f e} / 2}\left[N_{0}+G_{s}^{m}\left(f+m \Delta f_{p}\right)+G_{q}^{k m}\left(f+m \Delta f_{p}\right)\right] G_{p}^{m}\left(f+m \Delta f_{p}\right) \sin ^{2}\left(\pi f D T_{p}\right) d f}{\left|h_{p}^{m \leftarrow k m}\right|^{2} P_{p}^{k m}\left[2 \pi \int_{B_{0}-B_{f e} / 2}^{B_{0}+B_{f e} / 2} f G_{p}^{m}\left(f+m \Delta f_{p}\right) \sin \left(\pi f D T_{p}\right) d f\right]^{2}}
$$

$$
\left(\sigma_{\rho}^{k m}\right)^{2} \approx \frac{a T_{p}^{2}}{2}\left[\frac{1}{B_{f e} T_{p}\left(C / N_{0}\right)^{k m}}+\frac{B \sum_{k^{\prime} \in \mathcal{K}}(C P R)^{k m \leftarrow k^{\prime}}}{2 B_{f e}^{2}}+\frac{\sum_{k^{\prime} \in \mathcal{K}^{k}}(P P R)^{k m \leftarrow k^{\prime} m}}{B_{f e}^{2} T_{p}}\right]
$$

where $G_{c}^{n}(f)=T_{c} \operatorname{sinc}^{2}\left[\left(f-n \Delta f_{c}\right) T_{c}\right]$ is the normalized PSD of C-Sub $n . G_{q}^{k m}(f)$ is the PSD of the positioning components from other BSs which satisfies

$$
G_{q}^{k m}(f)=\sum_{k^{\prime} \in \mathcal{K}^{k}}\left|h_{p}^{m \leftarrow k^{\prime} m}\right|^{2} P_{p}^{k^{\prime} m} G_{p}^{m}(f)
$$

By taking (5), (7), (8) into (6) and using some approximations, (6) can be simplified to (9), where $C / N_{0}, C P R$ and $P P R$ are the carrier-to-noise ratio of positioning component, the equivalent communication-to-positioning ratio and the positioning-to-positioning ratio which are derived in Appendix $B$. The first item in (9) is caused by the noise, the second one is caused by the communication components from all BSs, and the third one is caused by the other BSs' positioning components. Because the positioning components are designed much weaker than communication ones in MS-NOMA, the interference from positioning components could be ignored. Then, (9) is further simplified to

$$
\begin{aligned}
\left(\sigma_{\rho}^{k m}\right)^{2} \approx & \frac{a T_{p}^{2}}{2}\left[\frac{1}{B_{f e} T_{p}\left(C / N_{0}\right)^{k m}}+\right. \\
& \left.\frac{B \sum_{k^{\prime} \in \mathcal{K}}(C P R)^{k m \leftarrow k^{\prime}}}{2 B_{f e}^{2}}\right]
\end{aligned}
$$

We define the ranging-factor $\left(\widetilde{\sigma}_{\rho}^{k m}\right)^{2}=\left(\sigma_{\rho}^{k m}\right)^{2} P_{p}^{k m}$ for later use. Notice that $\left(\widetilde{\sigma}_{\rho}^{k m}\right)^{2}$ has no relation to $P_{p}^{k m}$ which is a part of the denominator in (10).

\section{The Power Allocation of MS-NOMA WAVEForm}

\section{A. The Constraints}

1) The BER Threshold under QoS Constraint: To ensure the QoS of C-Users, the BERs of all C-Users should be limited under a certain threshold

$$
B E R^{k n} \leq \Xi_{t h}, \quad \forall k \in \mathcal{K}, \forall n \in \mathcal{N}
$$

Then, by taking (2) to (11) and rearranging items, we have

$$
\begin{aligned}
I^{k n} & \leq \frac{\gamma\left|h_{c}^{k n \leftarrow k n}\right|^{2} P_{c} T_{c}}{\operatorname{erfc}^{-1}\left(\Xi_{t h} / \Gamma\right)}-2 N_{0} \\
& \triangleq I_{t h}^{k n}, \quad \forall k \in \mathcal{K}, \forall n \in \mathcal{N}
\end{aligned}
$$

where $I_{t h}^{k n}$ is defined as the interference threshold of C-User $k n$, which is determined by the QoS requirement $\Xi_{t h}$.
2) The Total Power Limitation: The total transmit power is often limited. In MS-NOMA waveform, we have

$$
\sum_{m \in \mathcal{M}} P_{p}^{k m}+N P_{c} \leq P_{\mathrm{T}}^{k}, \forall k \in \mathcal{K}
$$

where $P_{\mathrm{T}}^{k}$ is the total transmit power of BS $k$. Let's define the positioning power budget of $\mathrm{BS} k$ as $P_{t h}^{k}=P_{\mathrm{T}}^{k}-N P_{c}$, then we have

$$
\sum_{m \in \mathcal{M}} P_{p}^{k m} \leq P_{t h}^{k}, \forall k \in \mathcal{K}
$$

3) The Elimination of Near-far Effect: To ensure that PUsers could receive as many positioning signals as possible, the power of the received positioning signals from different BSs must satisfy

$$
\frac{\left|h_{p}^{m \leftarrow k m}\right|^{2} P_{p}^{k m}}{\left|h_{p}^{m \leftarrow k^{\prime} m}\right|^{2} P_{p}^{k^{\prime} m}} \geq \varrho \Omega, \quad \forall m \in \mathcal{M}, \forall k \in \mathcal{K}, \forall k^{\prime} \in \mathcal{K}^{k}
$$

where $\Omega$ is the auto-correlation to cross-correlation ratio of positioning component, which is determined by the pseudorandom code and its length. $\varrho$ is determined by the receiver's performance, which is usually larger than 1 . For a particular P-Sub $k m$, if the strongest cross-correlation satisfies (15), all $k^{\prime} \mathrm{s}$ in (15) will be satisfied. Therefore, (15) can be rewritten as

$$
\left|h_{p}^{m \leftarrow k m}\right|^{2} P_{p}^{k m} \geq \varrho \Omega\left|h_{p}^{m \leftarrow k_{k}^{\prime} m}\right|^{2} P_{p}^{k_{k}^{\prime} m}, \forall m \in \mathcal{M}, \forall k \in \mathcal{K}
$$

where $k_{k}^{\prime} m$ represents the index of the strongest sub-carrier except the P-Sub $k m$ received by the P-User $m$.

\section{B. The Proposed Joint Power Allocation Model}

Our goal is to obtain the best positioning performance for all P-Users in terms of both accuracy and coverage under QoS requirement and total transmit power budget. So, the average lower bound of horizontal positioning error ${ }^{1}$ for all P-Users in the network is minimized by finding the optimal power values $P_{p}^{k m}, \forall m \in \mathcal{M}, \forall k \in \mathcal{K}$ under the given constraints. Considering the fact that the maximum negative value of a convex function is equivalent to its minimum. Then, the power allocation problem can be formulated as a convex optimization problem as follows

\footnotetext{
${ }^{1}$ For calculation convenience, we use the square of the horizontal positioning accuracy, i.e. $\left(\Psi^{m}\right)^{2}$
} 


$$
\begin{array}{r}
\text { OP1 } \max _{P_{p}^{k m}}-\frac{1}{M} \sum_{m \in \mathcal{M}}\left(\Psi^{m}\right)^{2} \\
\text { s.t. } I^{k n} \leq I_{t h}^{k n}, \forall n \in \mathcal{N}, \forall k \in \mathcal{K} \\
\sum_{m \in \mathcal{M}} P_{p}^{m \leftarrow k m} \leq P_{t h}^{k}, \forall k \in \mathcal{K} \\
\left|h_{p}^{m \leftarrow k m}\right|^{2} P_{p}^{k m} \geq \varrho \Omega\left|h_{p}^{m \leftarrow k_{k}^{\prime} m}\right|^{2} P_{p}^{k_{k}^{\prime} m}, \\
\forall m \in \mathcal{M}, \forall k \in \mathcal{K}
\end{array}
$$

OP1 can be solved by the Lagrange duality method [25]. Then, the Lagrange dual function of OP1 is then given by

$$
g(\mu, \nu, \beta)=\max _{P_{p}^{k m}} \mathcal{L}\left(\left\{P_{p}^{k m}\right\}, \mu, \nu, \beta\right)
$$

where $\mathcal{L}\left(\left\{P_{p}^{k m}\right\}, \mu, \nu, \beta\right)$ is the Lagrangian of $\mathrm{OP} 1, \mu=$ $\left\{\mu^{k n}\right\} \succeq 0, \nu=\left\{\nu^{k}\right\} \succeq 0$ and $\beta=\left\{\beta^{k m}\right\} \succeq 0$ are the matrices of dual variables associated with the corresponding constraints given in (12), (14) and (15). Then, the dual optimization problem can be formulated as

$$
\begin{aligned}
& \min g(\mu, \nu, \beta) \\
& \text { s.t. } \mu \succeq 0, \nu \succeq 0, \beta \succeq 0
\end{aligned}
$$

It is easy to be proved that $\mathcal{L}\left(\left\{P_{p}^{k m}\right\}, \mu, \nu, \beta\right)$ is linear in $\mu, \nu, \beta$ for fixed $P_{p}^{k m}$, and $g(\mu, \nu, \beta)$ is the maximum of linear function. Thus, the dual optimization problem is always convex. To solve this problem, the dual decomposition method introduced in [26] is employed. For this purpose, we introduce a transformation $\sum_{n \in \mathcal{N}}=\sum_{m \in \mathcal{M}} \sum_{n \in \mathbb{N}_{m}}$ to decompose the Lagrange dual function to $K \times M$ independent sub-problems, where

$$
\mathbb{N}_{m}=\{(2 G-1)(m-1)+1, \ldots,(2 G-1) m\}
$$

Then, we have

$$
\begin{aligned}
g(\mu, \nu, \beta) & =\sum_{k \in \mathcal{K}}\left[g^{k}(\mu, \nu, \beta)\right] \\
& =\sum_{k \in \mathcal{K}}\left\{\sum_{m \in \mathcal{M}} g^{k m}(\mu, \nu, \beta)+\nu^{k} P_{t h}^{k}\right\}
\end{aligned}
$$

where

$$
\begin{aligned}
& g^{k m}(\mu, \nu, \beta)= \max _{P_{p}^{k m}}\left\{-\frac{1}{M}\left(\lambda^{k m} \sigma_{\rho}^{k m}\right)^{2}-\nu^{k} P_{p}^{k m}\right. \\
&+\sum_{n \in \mathbb{N}_{m}} \mu^{k n}\left(I_{t h}^{k n}-I^{k n}\right) \\
&\left.+\beta^{k m}\left(\left|h_{p}^{m \leftarrow k m}\right|^{2} P_{p}^{k m}-\varrho \Omega\left|h_{p}^{m \leftarrow k_{k}^{\prime} m}\right|^{2} P_{p}^{k_{k}^{\prime} m}\right)\right\}
\end{aligned}
$$

From (26), it is clear that we can decompose the Lagrange dual function $g^{k}(\mu, \nu, \beta)$ to $M$ independent sub-problems by giving $\nu^{k}$. Each of the sub-problems is given by

$$
\begin{aligned}
& \text { OP2 } \max _{P_{p}^{k m}}-\frac{1}{M}\left(\lambda^{k m} \sigma_{\rho}^{k m}\right)^{2}-\nu^{k} P_{p}^{k m} \\
& \text { s.t. } I^{k n} \leq I_{t h}^{k n}, n \in \mathbb{N}_{m} \\
& \quad\left|h_{p}^{m \leftarrow k m}\right|^{2} P_{p}^{k m} \geq \varrho \Omega\left|h_{p}^{m \leftarrow k_{k}^{\prime} m}\right|^{2} P_{p}^{k_{k}^{\prime} m}
\end{aligned}
$$

Similar to OP1, the dual problem of the sub-problems can be expressed as

$$
\begin{aligned}
& \min \tilde{g}^{k m}\left(\tilde{\mu}^{k n}, \tilde{\beta}^{k m}\right) \\
& \text { s.t. } \tilde{\mu}^{k n} \geq 0, \forall n \in \mathbb{N}_{m} \\
& \tilde{\beta}^{k m} \geq 0
\end{aligned}
$$

where $\tilde{\mu}^{k n}$ and $\tilde{\beta}^{k m}$ are the non-negative dual variables for constraints (28) and (29), respectively.

The optimal power allocation solution $\tilde{P}_{p}^{k m}$ of OP2 can be obtained by using the Karush-Kuhn-Tucker (KKT) conditions as (33) shows, where $J^{k n \leftarrow m}$ can be found in Appendix C.

\section{The Positioning-Communication Joint Power Allocation Scheme}

The remaining task for solving OP1 is to obtain the optimal dual variables, which are the same in both OP1 and OP2. Applying the solution to OP2, we can obtain the optimal power allocation $\tilde{P}_{p}^{k m}$ in OP1. However, it is difficult to solve OP2 directly because we cannot obtain the closed-form expression of dual variables. The Lagrange dual function (25) is made up of $K$ independent sub-problems. For each sub-problem, it is observed that $\nu^{k}$ is the same for all P-Subs. $\mu^{k n}$ and $\beta^{k m}$ are different for C-Subs and P-Subs, respectively. Then, we can solve the optimization problem using the hierarchical algorithm by updating the values of the dual variables $\{\mu, \nu, \beta\}$ via subgradient methods, which guarantees the gradient-type algorithm to converge to the optimal solution [27].

Notice that the positions of P-Users are unknown which are necessary for calculating the geometric-dilution $\lambda^{k m}$. We can minimize the ranging error variance for all P-Users without considering the impact of the geometric-dilution at the first iteration. Namely, $\lambda^{k m}$ in (1) and (33) is set to 1 in the first fix. Then, we can get approximate positions and the initial allocated power. After several iterations, the positions and the power will converge to the optimal values.

The algorithm to solve OP1 can be summarized as Algorithm 1 shows. Where $t$ and $t^{\prime}$ are the iteration numbers. iter $N$ is the maximum iteration amount. $b_{1}, b_{2}$ and $b_{3}$ are the update step sizes. $\varepsilon>0$ is a given small constant.

\section{The Performance Evaluation}

In this section, we evaluate the communication performance, resource consumption and ranging accuracy of the proposed MS-NOMA waveform under a single-cell network firstly. Then, we examine the positioning performance in a 4-BSs cell by considering the impact of all factors comprehensively. 


$$
\tilde{P}_{p}^{k m}=\underbrace{\lambda^{k m}}_{\text {geometric-dilution }} \times \underbrace{\tilde{\sigma}_{\rho}^{k m}}_{\text {ranging-factor }} \times \underbrace{\left[M\left(\tilde{\beta}^{k m}\left|h_{p}^{m \leftarrow k m}\right|^{2}-\nu^{k}-\sum_{n \in \mathbb{N}_{m}} \tilde{\mu}^{k n} J^{k n \leftarrow m}\right)\right]^{-1 / 2}}_{\text {constraint-scale }}
$$

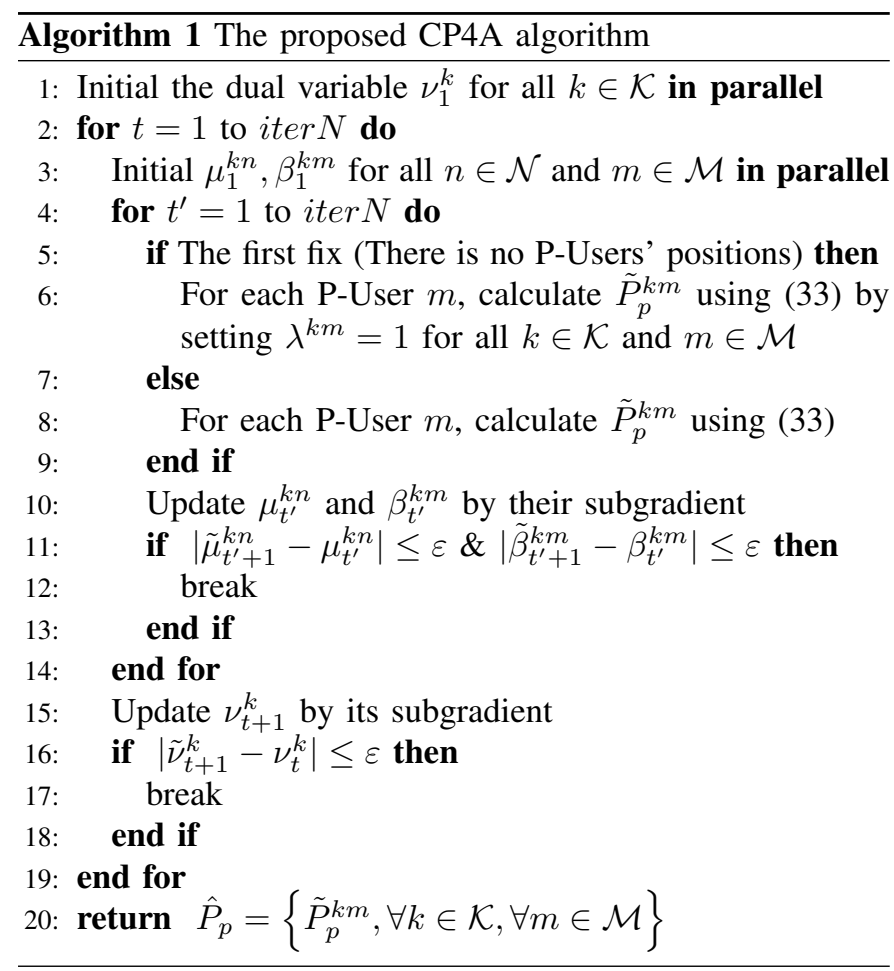

In the single-cell scenario, the impact of the cross-correlation will be vanished and the channel gains of the C-Subs and PSubs will be equal. So, the channel gains and index $k$ in (2) and (10) can be omitted. Then, we have

$$
\begin{gathered}
B E R^{n}=\Gamma \operatorname{erfc}\left(\frac{\gamma P_{c} T_{c}}{I^{n}+2 N_{0}}\right) \\
\left(\sigma_{\rho}^{m}\right)^{2} \approx \frac{a T_{p}^{2}}{2}\left[\frac{1}{B_{f e} T_{p}\left(C / N_{0}\right)^{m}}+\frac{B(C P R)^{m}}{2 B_{f e}^{2}}\right]
\end{gathered}
$$

where

$$
\begin{gathered}
I^{n}=\sum_{m=1}^{M} P_{p}^{m} T_{p} \operatorname{sinc}^{2}\left(m-\frac{n}{G}\right) \\
\left(C / N_{0}\right)^{m}=P_{p}^{m} / N_{0} \\
(C P R)^{m}=2 G P_{c} / P_{p}^{m}
\end{gathered}
$$

Notice that (34) and (35) reflect the features of MS-NOMA waveform itself without interference from other BSs.

The simulation settings are: The communication and positioning components use QPSK and BPSK constellation, respectively. The carrier frequency is set to $3.5 \mathrm{GHz}$ and $\Delta f_{c}=30 \mathrm{kHz}$. Two scenarios with different total bandwidths are considered: 1) $B=20 \mathrm{MHz}$ 2) $B=50 \mathrm{MHz}$. The amount of P-User is $M=20$. The power of all C-Users are assumed to be identical. The front-end bandwidth is set as twice of the total bandwidth, i.e. $B_{f e}=2 B$. The loop parameters are set as: $B_{L}=0.2 \mathrm{~Hz}, T_{\text {coh }}=0.02 \mathrm{~s}$ and $D=0.02$ chips.

\section{A. Communication Performance}

We first examine the interference of the positioning component to the C-Users. Fig. 3a shows the average BERs over the whole bandwidth when $P_{p}^{m}=P_{p}, \forall m \in \mathcal{M}$. It is clear that the average BERs decrease when $E_{b} / N_{0}$ and $C / N_{0}$ increase ( $E_{b}=P_{c} T_{c}$ is the energy of the communication symbol). Notice that the BER curves with small CPR will tend to be flat when $E_{b} / N_{0}$ becomes larger. This is because the interference caused by the positioning component dominates the BER performance rather than the environment noise (i.e. $I^{n}$ is much larger than $2 N_{0}$ ). When the positioning component becomes weaker (CPR becomes larger), the BER curves will become flat with larger $E_{b} / N_{0}$ and they will become closer to the one that only exists noise $(\mathrm{CPR}=\infty)$.

Fig. 3b takes an example of BERs over C-Subs in detail. If the power of the P-Subs is identical, the BERs are approximately identical as well. While the BERs are different when the power of P-Subs is allocated to different values. Then, the maximum BER is related to all P-Subs' power (see (34)) in this case. Therefore, the power of P-Subs must be allocated carefully to ensure the QoS of each C-Sub. Unsurprisingly, the lowest BERs are obtained when there are no positioning components.

\section{B. Resource Consumption of Positioning}

No matter what type of communication-positioning integration signal is used, the exclusive positioning waveform consumes the resources which should belong to communication. It is important that positioning consumes as few resources as possible to reduce the influence on communication. In this subsection, we compare the resource element and energy consumption between MS-NOMA and PRS. Some necessary parameters for this evaluation are defined as follows:

Define $E_{c}$ and $E_{p}$ as the energy of communication and positioning components in a unit time, respectively. $E_{\text {total }}=$ $E_{c}+E_{p}$ as the total energy. $T_{\text {meas }}$ as the measuring period which means the receiver executes measuring every $T_{\text {meas }}$ seconds. The positioning results update every $T_{\text {meas }}$ seconds as well.

For MS-NOMA, because it is continuous, its unit energy $E_{c}$ and $E_{p}$ are just equal to its power $N P_{c}$ and $M P_{p}$. For PRS, $P_{p}$ can be as strong as $P_{c}$. However, because PRS 


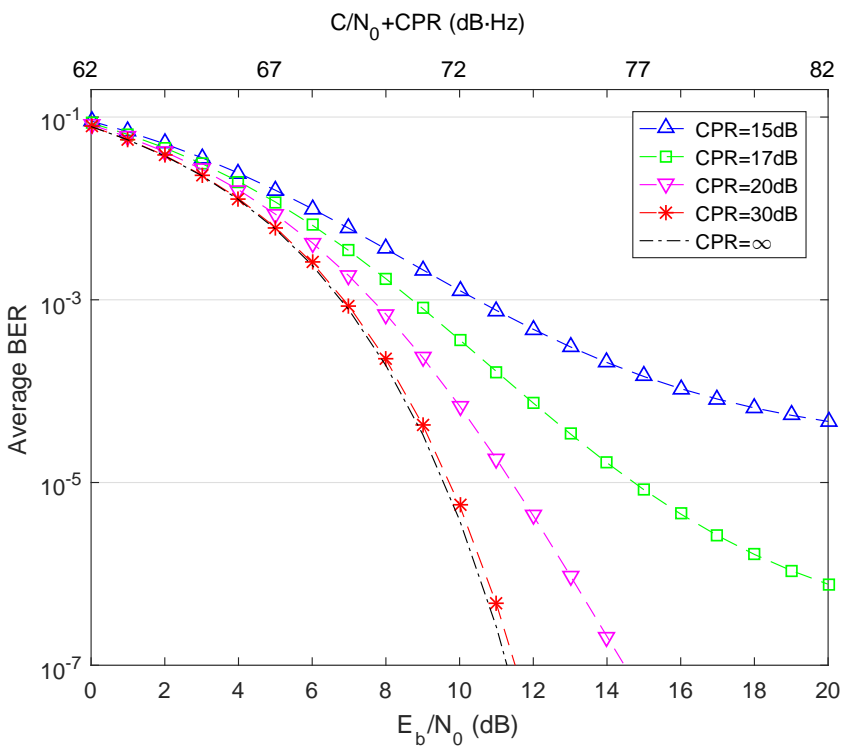

(a) Average BER

Fig. 3. Communication Performance

is discontinuous, its energy is determined by the amount of resource elements:

$$
\begin{aligned}
\left(E_{c} / E_{p}\right)_{P R S} & =N_{R E, c o m} / N_{R E, P R S} \\
& E_{c} \gg E_{p} \\
& \approx R E, \text { total } \\
& N_{R E, P R S}
\end{aligned}
$$

where $N_{R E}$ is the amount of resource elements during each measuring period, subscript com and total mean the communication component and the communication $+P R S$ components, respectively. To obtain the same spreading gains between MSNOMA and PRS for comparison, their code lengths should be identical during the integration time. So,

$$
N_{R E, P R S}=\Delta f_{p} T_{c o h}
$$

Notice that,

$$
N_{R E, \text { total }}=N T_{\text {meas }} / T_{c}
$$

Then,

$$
\left(E_{c} / E_{p}\right)_{P R S}=\frac{N}{G} \frac{T_{\text {meas }}}{T_{\text {coh }}}
$$

1) The resource element consumption: Although the positioning waveform of MS-NOMA does not occupy the resource elements directly, the extra BER loss produced by the positioning waveform could be seen as occupying the resource elements which should belong to communication. Therefore, (43) is introduced to evaluate its equivalent resource element consumption

$$
\Lambda_{M S-N O M A}=\frac{1}{N} \sum_{n} B E R^{n}-B E R_{0}
$$

where $B E R_{0}$ is the BER without positioning component. Then, by replacing $P_{c}$ and $P_{p}$ with $E_{c}$ and $E_{p}$ in (43), we can obtain the relationship between $\Lambda_{M S-N O M A}$ and the energy.

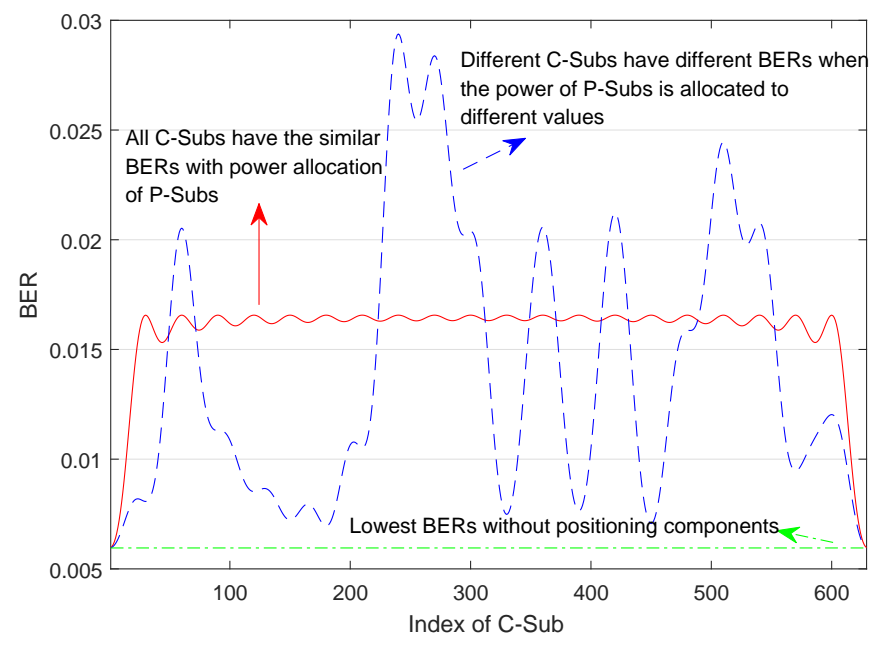

(b) Example of BERs over C-Subs $\left(E_{b} / N_{0}=5 \mathrm{~dB}, C P R=15 \mathrm{~dB}\right)$

PRS occupies the resource elements directly, its resource element consumption can be evaluated by

$$
\Lambda_{P R S}=\frac{N_{R E, P R S}}{N_{R E, \text { total }}}=\left(\frac{N}{G} \frac{T_{\text {meas }}}{T_{\text {coh }}}\right)^{-1}
$$

Fig. 4a shows the comparison of the resource element consumption between MS-NOMA and PRS. It is clear that MS-NOMA consumes fewer resource elements than PRS in most areas. This means although the positioning waveform interferes the communication as Section V-A analyzed, the equivalent resource element consumption is still smaller than PRS. Moreover, when the bandwidth increases, MS-NOMA consumes fewer resource elements because of the less positioning interference as (36) shows ( $T_{p}$ decreases). When $E_{\text {total }}$ increases, $\Lambda_{M S-N O M A}$ increases slightly. This is because the $B E R_{0}$ decreases slightly when the power of communication component increases. For comparison, $\Lambda_{P R S}$ has no relation to $B$ or $E_{\text {total }}$. This is because neither of them have impact on the proportion of communication and positioning components in PRS.

On the other hand, $\Lambda_{P R S}$ increases significantly when $T_{\text {meas }}$ decreases. This is unsurprising because it needs more PRS frames to ensure frequent measuring which consumes a great number of resource elements. On the contrary, the resource element consumption of MS-NOMA has no relation to $T_{\text {meas }}$. This is because MS-NOMA waveform is continuous, receiver could do measuring at any time within its calculation capacity. Therefore, PRS can hardly satisfy the frequent positioning scenarios like automatic driving.

2) The energy consumption: By giving certain $E_{\text {total }}$ and $E_{c} / E_{p}, P_{c}$ and $P_{p}$ can be determined as analyzed above. Then, the ranging accuracy is compared when MS-NOMA and PRS consume the same energy as Fig. 4b shows. Where the lower bound of PRS is used as introduced in [28]. It is 


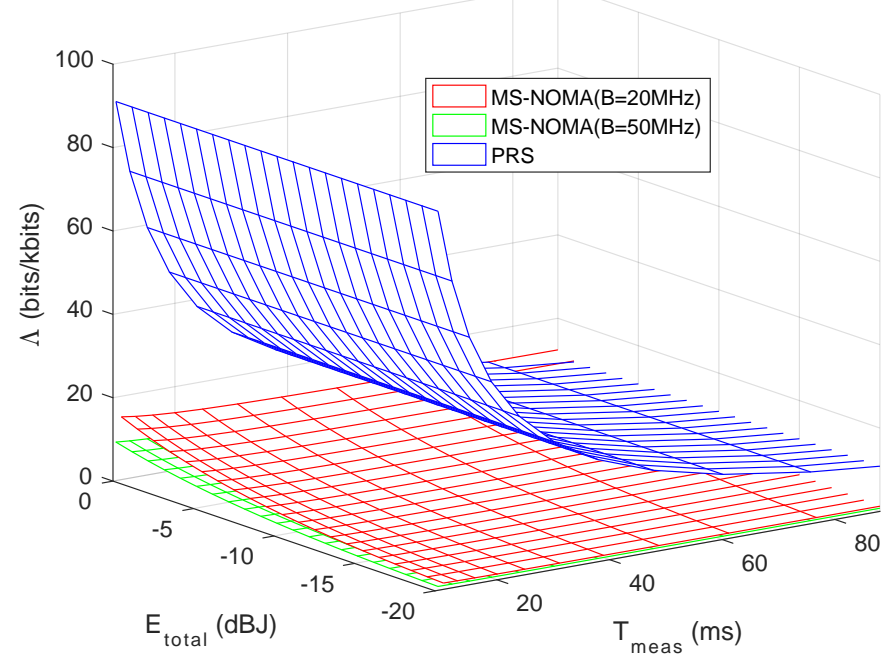

(a) The resource elements consumption

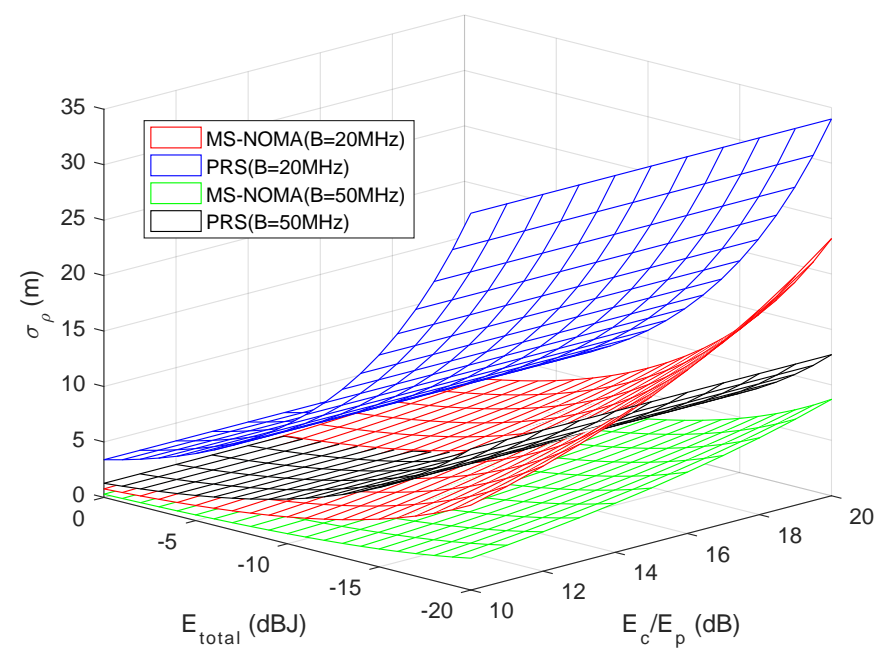

(b) The energy consumption

Fig. 4. Comparison of the positioning resource consumption between MS-NOMA and PRS

clear that MS-NOMA always has higher ranging accuracy than PRS under the same energy and bandwidth. Also, it is unsurprising that both two kinds of waveform have better ranging accuracy when they consume more energy. Moreover, when the proportion of positioning component decreases (i.e. $E_{c} / E_{p}$ increases), the ranging accuracy of MS-NOMA decreases gradually. This is because the interference of the communication component increases gradually. For comparison, the ranging accuracy of PRS does not change when $E_{c} / E_{p}$ changes. This is because PRS and communication waveform are TDMA which means they usually have the same power and different periods when $E_{c} / E_{p}$ changes. Therefore, the ranging accuracy will not change when the power keeps constant. Even so, the ranging accuracy of MS-NOMA is still better than PRS when the communication energy is 100 times higher than the positioning one.

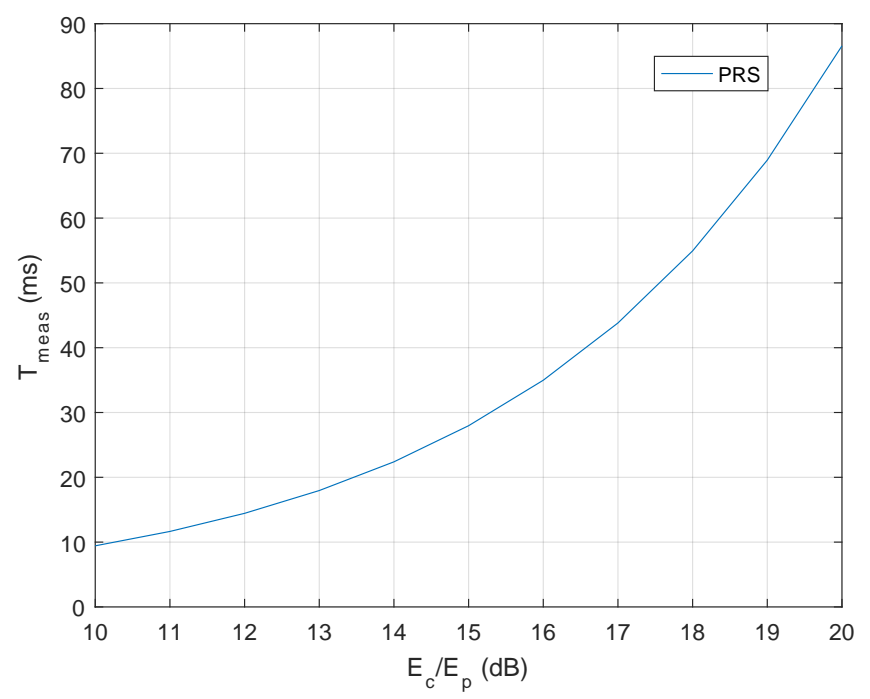

Fig. 5. The relationship between $T_{m e a s}$ and $E_{c} / E_{p}$ in PRS
On the other hand, although the ranging accuracy of PRS does not decrease when $E_{c} / E_{p}$ increases, higher $E_{c} / E_{p}$ results in longer duration of communication component. Then, it results in lower measuring frequency. The relationship between $T_{\text {meas }}$ and $E_{c} / E_{p}$ in PRS is shown in Fig. 5. It is clear that higher measuring frequency consumes more energy for positioning purpose (i.e. lower $E_{c} / E_{p}$ ) in PRS. For comparison, higher measuring frequency does not consume more energy in MS-NOMA.

\section{Positioning Performance}

For MS-NOMA, although stronger positioning power will have higher measurement accuracy, the maximum power of the positioning components will be limited by the QoS of communication as Fig. 3 shows. So, $P_{p}^{m}$ s must be allocated carefully to acquire the best ranging performance under the QoS constraint. In real applications, the communication interference from other BSs must be considered. In this subsection, we present the numerical results to evaluate the positioning performance of MS-NOMA waveform by using the proposed $\mathrm{CP} 4 \mathrm{~A}$ algorithm. The $\mathrm{BSs}$ are fixed at $(0,0)$, $(200,0),(200,200),(0,200)$ and 20 P-Users are randomly distributed in the coverage area. The free space propagation model is employed with 50 Monte Carlo runs in each simulation. Without any loss of generality, the parameters are set as bellow: $N P_{c}=1 \mathrm{~W}, P_{t h}^{k}=P_{t h}=0.1 \mathrm{~W}$ for any $k \in \mathcal{K}$. Then, $P_{T}^{k}=P_{T}=1.1 \mathrm{~W} . \Xi_{t h}=10^{-2}$ and $\varrho=2$.

Fig. 6 shows the positioning accuracy and coverage of MSNOMA waveform by using the proposed CP4A algorithm and the traditional equal-power transmission strategy, respectively. The positioning error is set to 0 if the P-User cannot receive more than 3 BSs which means there are not enough BSs for positioning. From Fig. 6a, we can see all P-Users have positioning results which means the near-far effect is dramatically reduced. While from Fig. 6b, it is clear that a great amount of P-Users $(50.8 \%)$ do not have positioning results by the traditional method as suffering severe near-far effect. 


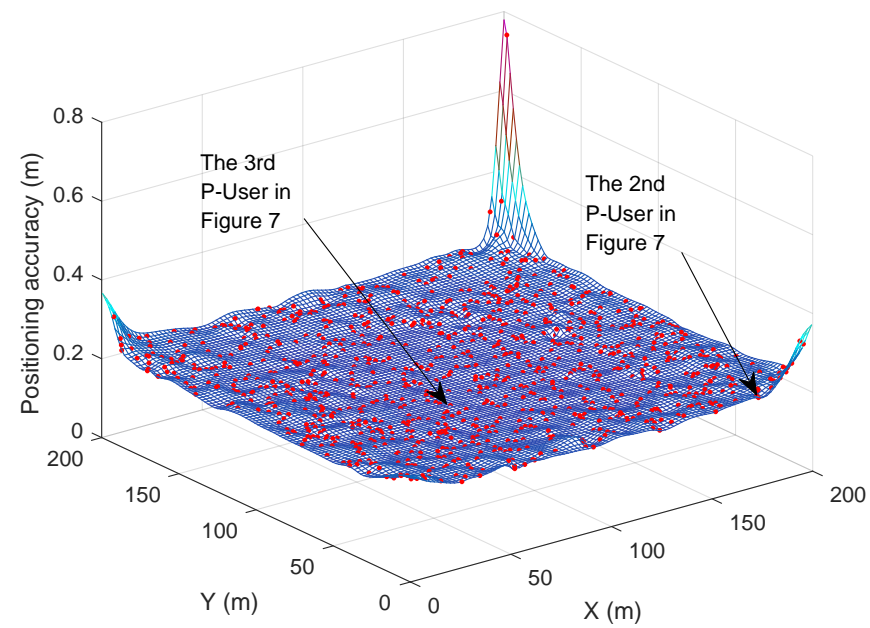

(a) The proposed CP4A algorithm

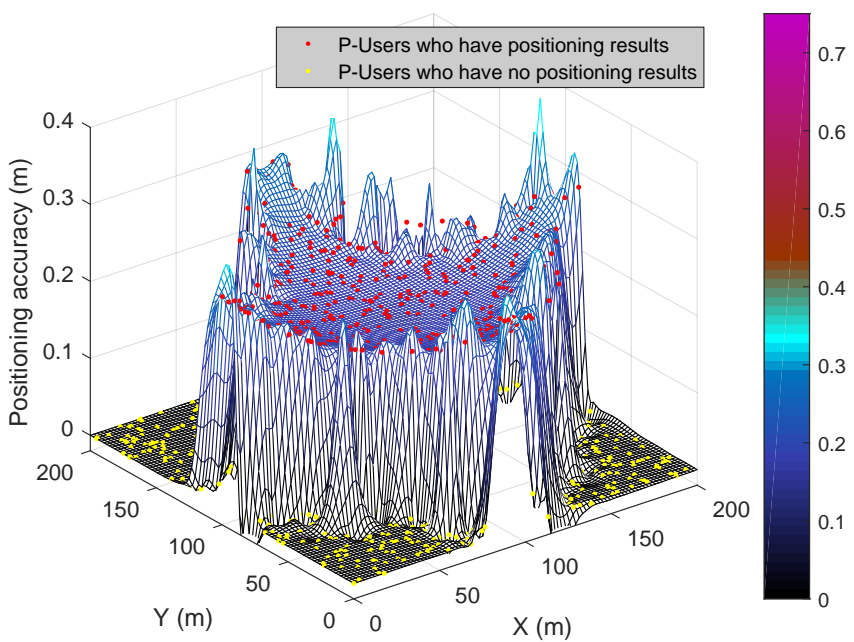

(b) The traditional method without power allocation

Fig. 6. The positioning coverage and accuracy of MS-NOMA with different power allocation algorithms $(B=50 \mathrm{MHz})$
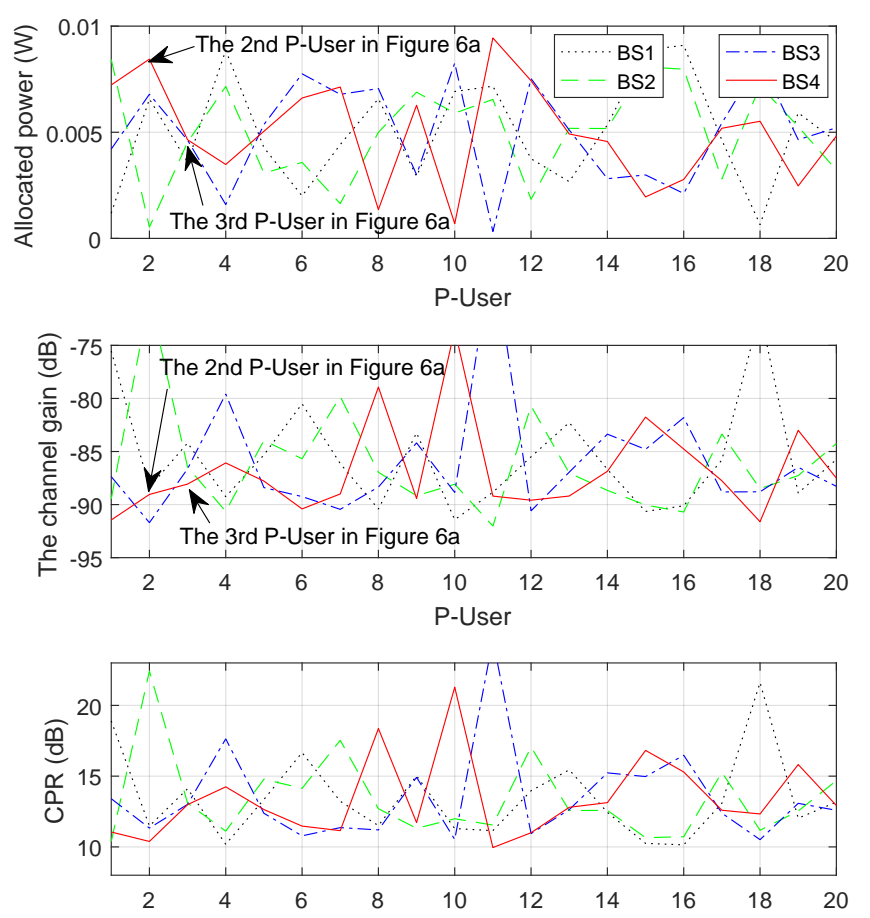

Fig. 7. The allocated power and CPRs with different channel gains $(B=$ $50 \mathrm{MHz})$

Fig. 7 illustrates the relationship between the allocated power, the channel gains $\left(\left|h_{p}^{m \leftarrow k m}\right|^{2}\right)$ and the CPRs of the P-Users by examining one simulation. It shows that the worse channel states tend to allocate stronger positioning power. This is exactly what we expected that the P-Users with worse channel states need stronger power to obtain an accurate ranging. However, notice that there is a power disparity between the $2^{\text {nd }}$ and $3^{\text {rd }}$ P-Users whose channel gains are similar. This is because the geometric-dilution $\lambda$ in (1) also affects the positioning accuracy which is considered by the power allocation process. This can be observed in Fig. 6a as well: The location of the $2^{\text {nd }} \mathrm{P}$-User (coordinate: $(178,8)$ ) is at the edge of the area compared to the center location of the $3^{\text {rd }}$ P-User (coordinate: $(79,77)$ ), so the $2^{\text {nd }} \mathrm{P}$-User has a stronger allocated power because of its bad geometric-dilution.

Moreover, it is obvious that CPRs have similar tends with the channel gains. It does not seem correct as CPR is inversely proportional to $\left|h_{p}^{m \leftarrow k m}\right|^{2}$ as defined in Appendix B. Notice that worse channel states tend to allocate stronger positioning power. As a result, the denominator of CPR (i.e. $\left|h_{p}^{m \leftarrow k m}\right|^{2} P_{p}^{k m}$ ) will tend to be flat for all $k m$. Then, CPR is approximately proportional to $\left|h_{c}^{m \leftarrow k^{\prime}}\right|^{2}$. Because the channel gains of communication components are approximately equal to that of positioning components for P-User $m$ (i.e. $\left|h_{c}^{m \leftarrow k^{\prime}}\right|^{2} \approx\left|h_{p}^{m \leftarrow k m}\right|^{2}$ ), it results in that CPRs have similar tends with $\left|h_{p}^{m \leftarrow k m}\right|^{2}$. In addition, it is observed that the CPRs have weaker fluctuations than the channel gains, this reflects that the proposed $\mathrm{CP} 4 \mathrm{~A}$ algorithm weakens the effects of channel attenuation.

TABLE II

THE COMPARISON BETWEEN MS-NOMA AND PRS

\begin{tabular}{|l|c|c|c|}
\hline \multicolumn{2}{|c|}{ Signal } & $\begin{array}{c}\text { MS-NOMA } \\
\text { (with CP4A) }\end{array}$ & PRS \\
\hline $\begin{array}{l}\text { Average positioning } \\
\text { accuracy }\end{array}$ & $20 \mathrm{MHz}$ & $0.57 \mathrm{~m}$ & $8.11 \mathrm{~m}$ \\
\cline { 2 - 4 } & $50 \mathrm{MHz}$ & $0.21 \mathrm{~m}$ & $3.28 \mathrm{~m}$ \\
\hline
\end{tabular}

The average positioning accuracy of MS-NOMA and PRS is shown in Table II. It shows the positioning accuracy of MS-NOMA waveform is much higher than that of PRS. Specifically, the improvements of MS-NOMA waveform are $93.0 \%$ and $93.6 \%$ compared to PRS with $20 \mathrm{MHz}$ and $50 \mathrm{MHz}$ bandwidth, respectively. Detailed comparisons are presented in Fig. 8a. Notice that the positioning accuracy represents the lower bound calculated by (1), so the minimum positioning error in Fig. 8a does not start from 0 . We can see nearly all of the error bounds of $20 \mathrm{MHz}$ MS-NOMA waveform are even 


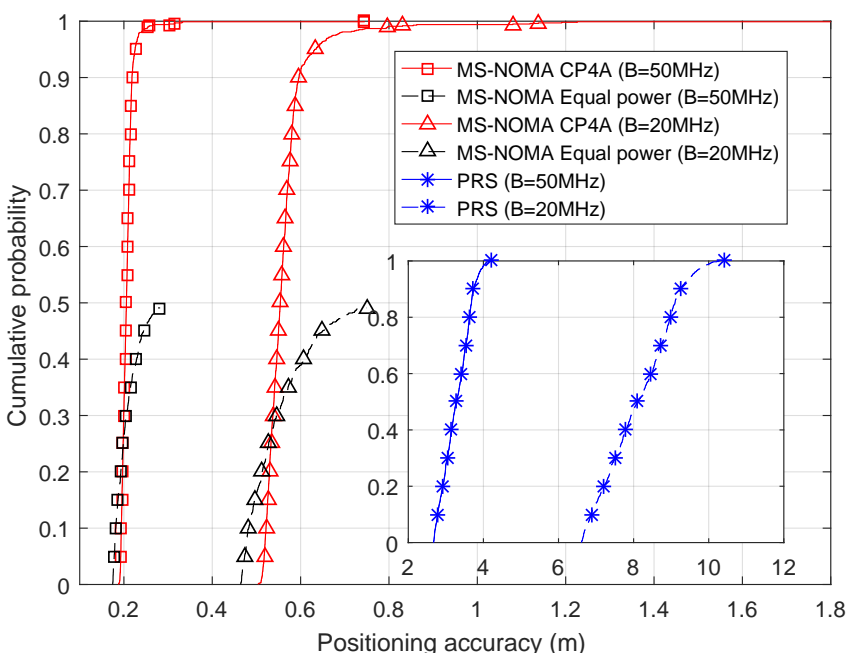

(a) The cumulative distribution functions (CDF) of different positioning schemes (The CDFs of MS-NOMA with equal power strategy approximately end at 0.5 as about $50 \%$ P-Users have no positioning results as Fig. $6 \mathrm{~b}$ shows)

Fig. 8. Detailed illustration of the positioning accuracy

smaller than the minimum error bound of $50 \mathrm{MHz}$ PRS, this is mainly because of the following two reasons:

1) Most of the ranging error variances of MS-NOMA are smaller than those of PRS as shown in Fig. 4b;

2) The largest ranging error amount all BSs will worsen the positioning error no matter how small the others are (see 1). So the positioning error bounds of PRS in peripheral area are higher than those in internal area as Fig. 8b shows (Larger path-loss results in larger ranging error variance). Moreover, the geometric-dilution $\lambda$ in peripheral area is usually larger than internal area which will further worsen the positioning accuracy. On the contrary, the proposed CP4A algorithm considers the ranging errors and geometric-dilution jointly. It averages the ranging errors weighted by the geometricdilution via configuring the positioning power of MS-NOMA waveform. Therefore, the positioning accuracy of MS-NOMA are flatter than those of PRS as compared between Fig. 6a and Fig. 8 b.

\section{CONCLUSIONS}

In this paper, we presented a feasibility study for a novel multiple access technique called Multi-Scale NonOrthogonal Multiple Access (MS-NOMA) for the next generation communication-positioning integration system. The MSNOMA waveform superposes power configurable positioning components on the communication ones to achieve high ranging accuracy and little resource consumption. We analyzed the BER for communication and the ranging accuracy for positioning when the two kinds of components exist simultaneously. The results show the interaction is rather limited and the proposed MS-NOMA waveform greatly improves the ranging accuracy comparing to the traditional PRS waveform. Next, the resource elements and energy consumption were analyzed. It is approved that the proposed MS-NOMA consumes much

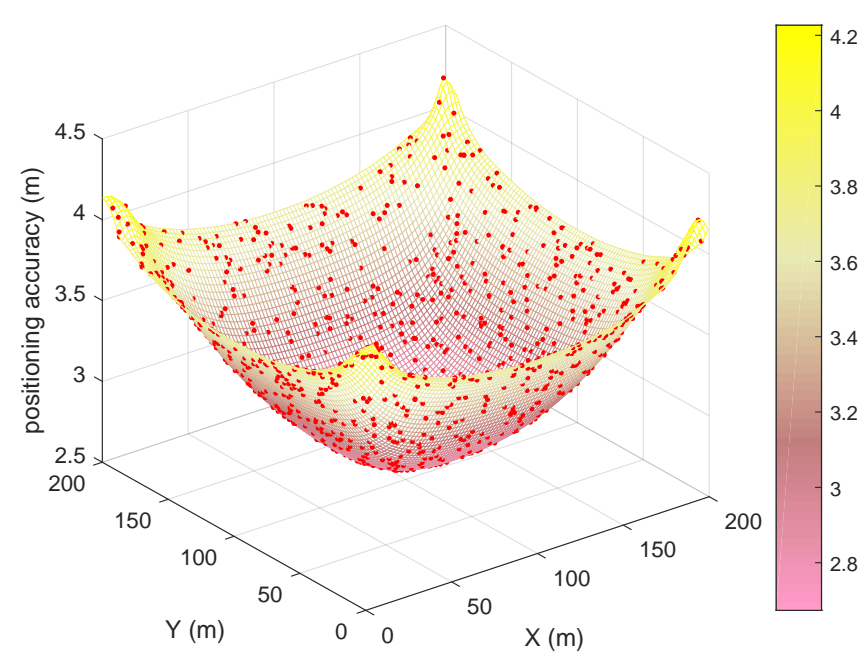

(b) The positioning accuracy of PRS $(B=50 \mathrm{MHz})$ fewer resources than PRS especially in frequent positioning scenarios like automatic driving. At last, we modeled a multi-user power allocation problem for optimal positioning accuracy and signal coverage as a convex optimization problem under the QoS requirement and other constraints. Then, a novel Communication and Positioning Performances constrained Positioning Power Allocation (CP4A) algorithm was proposed. The numerical results show our proposed MSNOMA waveform with CP4A algorithm improves the positioning accuracy and signal coverage dramatically comparing to the traditional PRS waveform.

\section{APPENDIX}

\section{A. Derivation of the Horizontal Positioning Accuracy}

Define $\varepsilon_{\rho}^{m}=\left[\varepsilon_{\rho}^{1 m}, \varepsilon_{\rho}^{2 m}, \ldots, \varepsilon_{\rho}^{k m}\right]^{T}$ as the ranging errors of P-User $m$, where $\varepsilon_{\rho}^{k m}$ represents the ranging error between BS $k$ and P-User $m$. Then, the positioning error of P-User $m$ is [29]

$$
\begin{aligned}
\varepsilon_{X}^{m} & =\left[\left(G^{m}\right)^{T} G^{m}\right]^{-1}\left(G^{m}\right)^{T} \varepsilon_{\rho}^{m} \\
& =H^{m} \varepsilon_{\rho}^{m}
\end{aligned}
$$

where

$$
\begin{gathered}
G^{m}=\left[\begin{array}{ccc}
\iota_{x}^{1 m} & \iota_{y}^{1 m} & \iota_{z}^{1 m} \\
\iota_{x}^{2 m} & \iota_{y}^{2 m} & \iota_{z}^{2 m} \\
\cdots & \cdots & \cdots \\
\iota_{x}^{k m} & \iota_{y}^{k m} & \iota_{z}^{k m}
\end{array}\right] \\
\left\{\begin{array}{c}
\iota_{x}^{k m}=\left(x_{p}^{m}-x_{b}^{k}\right) /\left\|X_{b}^{k}-X_{p}^{m}\right\| \\
\iota_{y}^{k m}=\left(y_{p}^{m}-y_{b}^{k}\right) /\left\|X_{b}^{k}-X_{p}^{m}\right\| \\
\iota_{z}^{k m}=\left(z_{p}^{m}-z_{b}^{k}\right) /\left\|X_{b}^{k}-X_{p}^{m}\right\|
\end{array}\right.
\end{gathered}
$$

where $X=[x, y, z]^{T}$ represents the coordinate. Subscript $p$ and $b$ represent P-User and BS, respectively. Because the ranging errors from the BSs are independent, their covariance 


$$
\begin{gathered}
A_{0}^{m}=\int_{B_{0}-B_{f e} / 2}^{B_{0}+B_{f e} / 2} f G_{p}^{m}\left(f+m \Delta f_{p}\right) \sin \left(\pi f D T_{p}\right) d f \\
A_{1}^{m}=\int_{B_{0}-B_{f e} / 2}^{B_{0}+B_{f e} / 2} N_{0} G_{p}^{m}\left(f+m \Delta f_{p}\right) \sin ^{2}\left(\pi f D T_{p}\right) d f \\
A_{2}^{m}=\int_{B_{0}-B_{f e} / 2}^{B_{0}+B_{f e} / 2} G_{s}^{m}\left(f+m \Delta f_{p}\right) G_{p}^{m}\left(f+m \Delta f_{p}\right) \sin ^{2}\left(\pi f D T_{p}\right) d f \\
A_{3}^{m}=\int_{B_{0}+B_{f e} / 2} G_{q}^{k m}\left(f+m \Delta f_{p}\right) G_{p}^{m}\left(f+m \Delta f_{p}\right) \sin ^{2}\left(\pi f D T_{p}\right) d f
\end{gathered}
$$

matrix is diagonal under the assumption that the range measuring is unbiased:

$$
\begin{aligned}
\left(\sigma_{\rho}^{m}\right)^{2} & =\operatorname{cov}\left(\varepsilon_{\rho}^{m}, \varepsilon_{\rho}^{m}\right) \\
& =\left[\begin{array}{cccc}
\left(\sigma_{\rho}^{1 m}\right)^{2} & 0 & \cdots & 0 \\
0 & \left(\sigma_{\rho}^{2 m}\right)^{2} & \cdots & 0 \\
\cdots & \cdots & \cdots & \cdots \\
0 & 0 & \cdots & \left(\sigma_{\rho}^{k m}\right)^{2}
\end{array}\right]
\end{aligned}
$$

where $\left(\sigma_{\rho}^{k m}\right)^{2}=\operatorname{cov}\left(\varepsilon_{\rho}^{k m}, \varepsilon_{\rho}^{k m}\right)$ represents the ranging error variance of P-Sub $\mathrm{km}$. Then, the covariance of the positioning error is

$$
\begin{aligned}
\left(\sigma_{X}^{m}\right)^{2} & =\operatorname{cov}\left(\varepsilon_{X}^{m}, \varepsilon_{X}^{m}\right) \\
& =H^{m}\left(\sigma_{\rho}^{m}\right)^{2}\left(H^{m}\right)^{T}
\end{aligned}
$$

The diagonal elements represent the positioning accuracy of each direction. Then, the horizontal positioning accuracy can be expressed as

$$
\Psi^{m}=\sqrt{\sum_{k \in \mathcal{K}}\left\{\left[\sum_{i=1}^{2}\left(\hbar_{i k}^{m}\right)^{2}\right]\left(\sigma_{\rho}^{k m}\right)^{2}\right\}}
$$

where $\hbar_{i k}^{m} \mathbf{s}(i \in\{1,2,3\})$ represent the elements of $H^{m}$. Note $\lambda^{k m}=\sqrt{\sum_{i=1}^{2}\left(\hbar_{i k}^{m}\right)^{2}}$ as the geometric-dilution, then we have (1).

\section{B. Derivation of $\left(\sigma_{\rho}^{k m}\right)^{2}$}

Note (51)-(54).

Then, (6) can be written as

$$
\left(\sigma_{\rho}^{k m}\right)^{2}=\frac{a\left(A_{1}^{m}+A_{2}^{m}+A_{3}^{m}\right)}{(2 \pi)^{2}\left|h_{p}^{m \leftarrow k m}\right|^{2} P_{p}^{k m}\left(A_{0}^{m}\right)^{2}}
$$

Notice that there are multiple P-Users, i.e. the bandwidth of the positioning waveform for one P-User is much smaller than the total bandwidth $B$. Moreover, the front-end bandwidth is larger than $B$ as well. So we have $B_{f e} \gg 2 / T_{p}$. Consequently, a DLL's narrow early-late spacing $D$ can be applied ${ }^{2}$. When $D \rightarrow 0, \sin \left(\pi f D T_{p}\right)$ in (51)-(54) can be replaced by Taylor expansion around 0 . Then, by taking (5), (7), (8) into (51)-(54) and rearranging items, we have (56)-(59).

Taking (56)-(60) back to (55) and rearranging items, we have (61), where

$$
\left|h_{c}^{m \leftarrow k^{\prime}}\right|^{2}=\frac{2}{N} \sum_{n \in \mathcal{N}}\left|h_{c}^{m \leftarrow k^{\prime} n}\right|^{2} \sin ^{2}\left(\frac{n}{G} \pi\right)
$$

is defined as the normalized equivalent channel gain of the communication component transmitted by BS $k^{\prime}$ to the P-User $m$.

Let's define $\left(C / N_{0}\right)^{k m}=\left|h_{p}^{m \leftarrow k m}\right|^{2} P_{p}^{k m} / N_{0}$ as the carrier-to-noise ratio of P-Sub $k m,(C P R)^{k m \leftarrow k^{\prime}}=$ $\frac{2 G\left|h_{c}^{m \leftarrow k^{\prime}}\right|^{2} P_{c}}{\left|h_{p}^{m \leftarrow k m}\right|^{2} P_{p}^{k m}}$ as the equivalent communication-to-positioning ratio of communication component transmitted by $\mathrm{BS} k^{\prime}$ to P-Sub $k m$, and $(P P R)^{k m \leftarrow k^{\prime} m}=\frac{\left|h_{p}^{m \leftarrow k^{\prime} m}\right|^{2} P_{p}^{k^{\prime} m}}{\left|h_{p}^{m \leftarrow k m}\right|^{2} P_{p}^{k m}}$ as the positioning-to-positioning ratio of P-Sub $k^{\prime} m$ to P-Sub $k m$. Then we have (9).

C. Derivation of $\tilde{P}_{p}^{k m}$

The KKT conditions of OP2 can be written as

$$
\begin{aligned}
\sum_{n \in \mathbb{N}_{m}} \tilde{\mu}^{k n}\left(I_{t h}^{k n}-I^{k n}\right) & =0 \\
\tilde{\beta}^{k m}\left(\left|h_{p}^{m \leftarrow k m}\right|^{2} P_{p}^{k m}-\varrho \Omega\left|h_{p}^{m \leftarrow k_{k}^{\prime} m}\right|^{2} P_{p}^{k_{k}^{\prime} m}\right) & =0 \\
\frac{\partial \tilde{\mathcal{L}}\left(\left\{P_{p}^{k m}\right\}, \tilde{\mu}^{k n}, \tilde{\beta}^{k m}\right)}{\partial P_{p}^{k m}} & =0
\end{aligned}
$$

It is obvious that the optimal solution $\tilde{P}_{p}^{k m}$ satisfies (65). Thus, (65) can be simplified to (66).

By taking (3) into (66), we have

$$
J^{k n \leftarrow m}=\sum_{k^{\prime} \in \mathcal{K}}\left|h_{p}^{k n \leftarrow k^{\prime} m}\right|^{2} T_{p} \operatorname{sinc}^{2}\left(m-\frac{n}{G}\right)
$$

${ }^{2}$ If $B_{f e}$ is not large enough, the DLL correlation peak will be flattened which will deteriorate the performance of the phase discriminator. 


$$
\begin{aligned}
& A_{0}^{m}=\pi D T_{p}^{2} \int_{-B_{f e} / 2}^{B_{f e} / 2} f^{2} \operatorname{sinc}^{2}\left(f T_{p}\right) d f=\frac{1}{2 \pi} D B_{f e} \\
& A_{1}^{m}=\pi D T_{p} N_{0} A_{0}^{m} \\
& A_{2}^{m}=D^{2} T_{p} \sum_{k^{\prime} \in \mathcal{K}} \sum_{n \in \mathcal{N}}\left|h_{c}^{m \leftarrow k^{\prime} n}\right|^{2} P_{c} T_{c} \int_{-B_{f e} / 2}^{B_{f e} / 2} \operatorname{sinc}^{2}\left[\left(f+m \Delta f_{p}-n \Delta f_{c}\right) T_{c}\right] \sin ^{2}\left(f T_{p}\right) d f \\
& \stackrel{G \gg 1}{\approx} D^{2} T_{p} T_{c} P_{c} \sum_{k^{\prime} \in \mathcal{K}} \sum_{n \in \mathcal{N}}\left|h_{c}^{m \leftarrow k^{\prime} n}\right|^{2} \sin ^{2}\left[\pi\left(m-\frac{n}{G}\right)\right] \times \int_{(G m-n-1) \Delta f_{c}}^{(G m-n+1) \Delta f_{c}} \operatorname{sinc}^{2}\left[\left(f+m \Delta f_{p}-n \Delta f_{c}\right) T_{c}\right] d f \\
& \approx D^{2} T_{p} P_{c} \sum_{k^{\prime} \in \mathcal{K}} \sum_{n \in \mathcal{N}}\left|h_{c}^{m \leftarrow k^{\prime} n}\right|^{2} \sin ^{2}\left(\frac{n}{G} \pi\right) \\
& A_{3}^{m}=\pi^{2} D^{2} T_{p}^{4} \sum_{k^{\prime} \in \mathcal{K}^{k}}\left|h_{p}^{m \leftarrow k^{\prime} m}\right|^{2} P_{p}^{k^{\prime} m} \underbrace{\int_{-B_{f e} / 2}^{B_{f e} / 2} f^{2} \operatorname{sinc}^{4}\left(f T_{p}\right) d f}_{\bar{A}_{3}}
\end{aligned}
$$

where

$$
\begin{aligned}
\bar{A}_{3}{ }^{B_{f e} \gg 2 / T_{p}} \int_{-\infty}^{\infty} \frac{\sin ^{4}\left(\pi f T_{p}\right)}{\pi^{4} f^{2} T_{p}^{4}} d f=\frac{1}{4 \pi^{4} T_{p}^{4}} \int_{-\infty}^{\infty}\left[\frac{4 \sin ^{2}\left(\pi f T_{p}\right)}{f^{2}}-\frac{\sin ^{2}\left(2 \pi f T_{p}\right)}{f^{2}}\right] d f=\frac{1}{2 \pi^{2} T_{p}^{3}} \\
\left(\sigma_{\rho}^{k m}\right)^{2} \approx \frac{a T_{p}}{2}\left[\frac{N_{0}}{B_{f e}\left|h_{p}^{m \leftarrow k m}\right|^{2} P_{p}^{k m}}+\frac{2 P_{c} \sum_{k^{\prime} \in \mathcal{K}} \sum_{n=1}^{N} \mid h_{c}^{\left.m \leftarrow k^{\prime} n\right|^{2}} \sin ^{2}\left(\frac{n}{G} \pi\right)}{B_{f e}^{2}\left|h_{p}^{m \leftarrow k m}\right|^{2} P_{p}^{k m}}+\frac{\sum_{k^{\prime} \in \mathcal{K}^{k}}\left|h_{p}^{m \leftarrow k^{\prime} m}\right|^{2} P_{p}^{k^{\prime} m}}{B_{f e}^{2}\left|h_{p}^{m \leftarrow k m}\right|^{2} P_{p}^{k m}}\right] \\
=\frac{a T_{p}^{2}}{2}\left(\frac{N_{0}}{B G P_{c} \sum_{k^{\prime} \in \mathcal{K}}\left|h_{c}^{m \leftarrow k^{\prime}}\right|^{2}} \sum_{B_{f e} T_{p}\left|h_{p}^{m \leftarrow k m}\right|^{2} P_{p}^{k m}}^{B_{f e}^{2}\left|h_{p}^{m \leftarrow k m}\right|^{2} P_{p}^{k m}}+\frac{\sum_{k^{\prime} \in \mathcal{K}^{k}}\left|h_{p}^{m \leftarrow k^{\prime} m}\right|^{2} P_{p}^{k^{\prime} m}}{B_{f e}^{2} T_{p}\left|h_{p}^{m \leftarrow k m}\right|^{2} P_{p}^{k m}}\right)
\end{aligned}
$$

$$
\begin{aligned}
\frac{\partial \tilde{\mathcal{L}}}{\partial P_{p}^{k m}} & =\frac{-\frac{1}{M} \partial\left(\lambda^{k m} \sigma_{\rho}^{k m}\right)^{2}-\nu^{k} P_{p}^{k m}}{\partial P_{p}^{k m}}+\frac{\partial \sum_{n \in \mathbb{N}_{m}} \tilde{\mu}^{k n}\left(I_{t h}^{k n}-I^{k n}\right)}{\partial P_{p}^{k m}}+\frac{\partial\left\{\tilde{\beta}^{k m}\left(\left|h_{p}^{m \leftarrow k m}\right|^{2} P_{p}^{k m}-\varrho \Omega\left|h_{p}^{m \leftarrow k_{k}^{\prime} m}\right|^{2} P_{p}^{k_{k}^{\prime} m}\right)\right\}}{\partial P_{p}^{k m}} \\
& =-\frac{1}{M}\left(\frac{\lambda^{k m} \tilde{\sigma}_{\rho}^{k m}}{P_{p}^{k m}}\right)^{2}-\nu^{k}-\sum_{n \in \mathbb{N}_{m}} \tilde{\mu}^{k n} \underbrace{\frac{\partial I^{k n}}{\partial P_{p}^{k m}}}_{J^{k n \leftarrow m}}+\tilde{\beta}^{k m}\left|h_{p}^{m \leftarrow k m}\right|^{2}
\end{aligned}
$$


Then, by setting (66) to 0 , we can obtain the optimal power allocation solution as (33) shows.

\section{REFERENCES}

[1] J. A. del Peral-Rosado, R. Raulefs, J. A. Lopez-Salcedo, and G. SecoGranados, "Survey of cellular mobile radio localization methods: from $1 \mathrm{G}$ to 5G," IEEE Communications Surveys and Tutorials, vol. 20, no. 2, pp. 1124-1148, 2018.

[2] N. Zhu, M. Ortiz, and V. Renaudin, "Seamless indoor-outdoor infrastructure-free navigation for pedestrians and vehicles with GNSSaided foot-mounted IMU," in 2019 International Conference on Indoor Positioning and Indoor Navigation (IPIN), 2019, pp. 1-8.

[3] L. Yin, Q. Ni, and Z. Deng, "A GNSS/5G integrated positioning methodology in D2D communication networks," IEEE Journal on Selected Areas in Communications, vol. 36, no. 2, pp. 351-362, 2018.

[4] Y. Tao and L. Zhao, "AIPS: An accurate indoor positioning system with fingerprint map adaptation," IEEE Internet of Things Journal, pp. 1-1, 2021.

[5] S. Han, Y. Li, W. Meng, C. Li, T. Liu, and Y. Zhang, "Indoor localization with a single Wi-Fi access point based on OFDM-MIMO," IEEE Systems Journal, vol. 13, no. 1, pp. 964-972, 2019.

[6] K. McDermott, R. M. Vaghefi, and R. M. Buehrer, "Cooperative UTDOA positioning in LTE cellular systems," in 2015 IEEE Globecom Workshops (GC Wkshps), 2015, pp. 1-6.

[7] Z. Chaloupka, "Technology and standardization gaps for high accuracy positioning in 5G," IEEE Communications Standards Magazine, vol. 1, no. 1, pp. 59-65, 2017.

[8] G. Kumar, V. Gupta, and R. Tank, "Phase-based angle estimation approach in indoor localization system using Bluetooth Low Energy," in 2020 International Conference on Smart Electronics and Communication (ICOSEC), 2020, pp. 904-912.

[9] B. Yang, X. Cao, K. Xiong, C. Yuen, Y. L. Guan, S. Leng, L. Qian, and Z. Han, "Edge intelligence for autonomous driving in $6 \mathrm{G}$ wireless system: Design challenges and solutions," IEEE Wireless Communications, vol. 28, no. 2, pp. 40-47, 2021.

[10] Y. Xianjia, L. Qingqing, J. P. Queralta, J. Heikkonen, and T. Westerlund, "Applications of UWB networks and positioning to autonomous robots and industrial systems," in 2021 10th Mediterranean Conference on Embedded Computing (MECO), 2021, pp. 1-6.

[11] C. Satya Ganesh Nutan Dev, L. Pathak, G. Ponnamareddy, and D. Das, "NRPos: A multi-RACH framework for 5G NR positioning," in 2020 IEEE 3rd 5G World Forum (5GWF), 2020, pp. 25-30.

[12] 3GPP, "TS 22.261 v17.0.1: Service requirements for the 5G system," 2019.

[13] J. Schloemann, H. S. Dhillon, and R. M. Buehrer, "A tractable analysis of the improvement in unique localizability through collaboration," IEEE Transactions on Wireless Communications, vol. 15, no. 6, pp. 3934 3948,2015

[14] C. H. Chen and K. T. Feng, "Enhanced distance and location estimation for broadband wireless networks," IEEE Transactions on Mobile Computing, vol. 14, no. 11, pp. 1-1, 2015.

[15] Z. Ding, X. Lei, G. K. Karagiannidis, R. Schober, J. Yuan, and V. K. Bhargava, "A survey on non-orthogonal multiple access for 5G networks: Research challenges and future trends," IEEE Journal on Selected Areas in Communications, vol. 35, no. 10, pp. 2181-2195, 2017.

[16] Y. Liu, Z. Qin, M. Elkashlan, G. Yue, and L. Hanzo, "Enhancing the physical layer security of non-orthogonal multiple access in large-scale networks," IEEE Transactions on Wireless Communications, vol. 16, no. 3, pp. 1656-1672, 2017.

[17] A. K. Karmokar, M. Naeem, and A. Anpalagan, "Energy-efficient subcarrier power allocation for cognitive radio networks using statistical interference model," in IEEE International Symposium on Personal, Indoor, and Mobile Radio Communications, 2015.

[18] X. Ding and Q. Li, "Joint power control and time allocation for wireless powered underlay cognitive radio networks," IEEE Wireless Communications Letters, vol. PP, no. 99, pp. 1-1, 2017.

[19] Y. Liu, Z. Ding, M. Elkashlan, and J. Yuan, "Nonorthogonal multiple access in large-scale underlay cognitive radio networks," IEEE Transactions on Vehicular Technology, vol. 65, no. 12, pp. 10 152-10157, 2016.

[20] Z. Ding, H. Dai, and H. V. Poor, "Relay selection for cooperative NOMA," IEEE Wireless Communications Letters, vol. 5, no. 4, pp. 416 419, 2016.
[21] L. Yin, J. Cao, K. Lin, Z. Deng, and Q. Ni, "A novel positioningcommunication integrated signal in wireless communication systems," IEEE Wireless Communications Letters, pp. 1-1, 2019.

[22] F. Maschietti, G. Fodor, D. Gesbert, and P. d. Kerret, "User coordination for fast beam training in FDD multi-user massive MIMO," IEEE Transactions on Wireless Communications, vol. 20, no. 5, pp. 29612976, 2021.

[23] Y. Liu, Z. Ding, M. Elkashlan, and H. V. Poor, "Cooperative nonorthogonal multiple access with simultaneous wireless information and power transfer," IEEE Journal on Selected Areas in Communications, vol. 34, no. 4, pp. 938-953, 2016.

[24] J. W. Betz and K. R. Kolodziejski, "Generalized theory of code tracking with an early-late discriminator Part I: Lower bound and coherent processing," IEEE Transactions on Aerospace and Electronic Systems, vol. 45, no. 4, pp. 1538-1556, 2009.

[25] Boyd, Vandenberghe, and Faybusovich, Convex Optimization, 2004

[26] R. Zhang, "Optimal power control over fading cognitive radio channel by exploiting primary user CSI," in IEEE Global Communications Conference (GLOBECOM), Nov 2008, pp. 1-5.

[27] L. Li and C. Xu, "On ergodic sum capacity of fading channels in OFDMA-based cognitive radio networks," IEEE Transactions on Vehicular Technology, vol. 63, no. 9, pp. 4334-4343, Nov 2014.

[28] J. A. del Peral-Rosado, J. A. Lopez-Salcedo, G. Seco-Granados, F. Zanier, and M. Crisci, "Achievable localization accuracy of the positioning reference signal of 3GPP LTE," in 2012 International Conference on Localization and GNSS, 2012, pp. 1-6.

[29] Y. Lu, Z. Deng, Z. Di, and E. Hu, "Quality assessment method of GNSS signals base on multivariate dilution of precision," in European Navigation Conference, 2016.

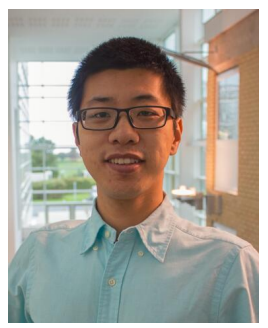

Lu Yin (M'17) received the B.Sc. and Ph.D degrees in electrical engineering from Beijing University of Posts and Telecommunications (BUPT), Beijing, China, in 2014. He is currently an associate professor with School of Electronic Engineering, BUPT. He was an academic visitor with Lancaster University from 2016 to 2017 . His current research interests are in the areas of GNSS, indoor positioning, integrated/cooperative positioning, intelligent localization (localization with artificial intelligence), positioning-communication integration system and communication system. He is now involved in the development and standardization of $3 \mathrm{GPP} 5 \mathrm{G}$ NR positioning.

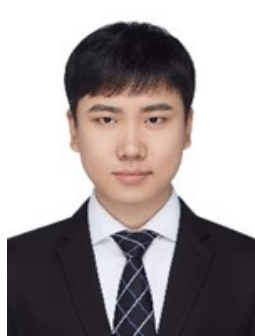

Jiameng Cao received the B.S. and M.S. degrees from Beijing University of Posts and Telecommunications, Beijing, China, in 2017 and 2020, respectively. His research interests are in the areas of wireless positioning, inertial navigation and communication systems. He is currently engaged in research on virtualization and docker container orchestration in ZTE Research Institution, Xi'an, China. 


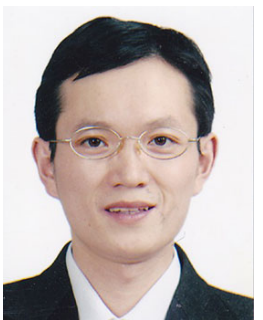

Qiang Ni (M'04-SM'08) received the B.Sc., M.Sc., and Ph.D. degrees from the Huazhong University of Science and Technology, China, all in engineering. $\mathrm{He}$ is currently a Professor and the Head of the Communication Systems Group, School of Computing and Communications, Lancaster University, InfoLab21, Lancaster, U.K. His main research interests lie in the area of future generation communications and networking, including green communications and networking, cognitive radio network systems, heterogeneous networks, small cell and ultra-dense networks, 5G and 6G, SDN, cloud networks, energy harvesting, wireless information and power transfer, IoTs and vehicular networks. He has authored or co-authored over 300 papers in these areas. He was the IEEE 802.11 Wireless Standard Working Group Voting Member and a contributor to the IEEE Wireless Standards.

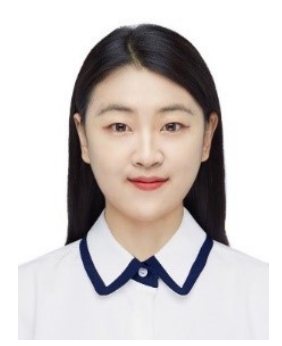

Yuzheng Ma received the B.S. degree from Jilin University, Jilin, China, in 2019. She is currently pursuing the M.S. degree in the Beijing University of Posts and Telecommunications, Beijing, China. Her current research interests are in the areas of wireless positioning and protocal for $5 \mathrm{G} \mathrm{NR}$ positioning.

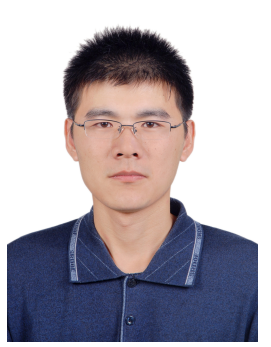

Song $\mathbf{L i}$ received the B.Sc. and M.Sc. degrees in communication and information system from Tianjin University, Tianjin, China, in 2007 and 2009, respectively, and the $\mathrm{Ph} . \mathrm{D}$. degree in signal and information processing from the Beijing University of Posts and Telecommunications, Beijing, China, in 2012. He was a visiting scholar with Lancaster University, UK from 2016 to 2017 . He is currently an associate professor with the School of Information and Control Engineering, China University of Mining and Technology, Xuzhou, China. His current research interests include B5G wireless networks, cyber-physical system, and industrial Internet of Things. 